\title{
Analysis of a Risk-Based Model for the Growth of AIDS Infection*
}

\author{
GARY DE YOUNG \\ Institute of Theoretical Dynamics, University of California, Davis, California 95616
}

PHII IP K. MAINI

Centre for Mathematical Biology, Mathematical Institute, Oxford, England OX1 3LB

AND

MICHAEL NAKAMAYE

Department of Mathematics, Yale University, New Haven, Connecticut 06520

Received 29 August 1990; revised 2 March 1991

\begin{abstract}
Several models for the spread of AIDS within a homosexual community have been proposed that incorporate biased mixing of different risk groups. A simple model is presented that captures many of the features of these more complex models. Analytical expressions are derived for the time to the state of maximum infection (SMI) in a particular risk group, the proportion infected at SMI, and the number of infected individuals as the group approaches SMI. These results agree qualitatively with numerical simulations of the model.
\end{abstract}

\section{INTRODUCTION}

The growth of the human immunodeficiency virus (HIV) and acquired immunodeficiency syndrome (AIDS) has been the subject of much recent theoretical and medical research (see, for example, [2-4, 8, 18, 20]). As more becomes known about AIDS, the mathematical models proposed to account for the spread of the disease become more complex. The 1988 paper by Hyman and Stanley [15] contains a review of some of the main processes involved and presents numerical simulations of several models that account for such factors as variable infectiousness of individuals and variable incubation periods in a homosexual community. Anderson et al. [5] presented a model for the spread of disease in the U.K. homosexual

\footnotetext{
*This paper was submitted while the authors were at the Department of Mathematics, University of Utah, Salt Lake City, Utah.
} 
population using field data and considered the effects of changes in sexual behavior. Anderson and coworkers $[6,19]$ analyzed a model for the spread of AIDS in heterosexual communities in developing countries and explored the effects of control strategies. Although the majority of AIDS cases in developed countries occur in the homosexual community, the disease is spreading into the heterosexual community. Hethcote [14] presented a general model that accounts for interaction between heterosexuals, bisexuals, homosexuals, and intravenous drug abusers.

Models of a homogeneous population in which behavior is constant with time predict that the initial growth of disease is exponential with constant growth rate. However, recent surveys show that the total number of AIDS cases reported to the Centers for Disease Control (CDC) in the United States has grown as the cube of time $[9,11,15,16]$, which implies that the growth rate is decreasing inversely with time. These data relate to AIDS patients infected before the publicity campaigns for AIDS prevention began; thus it is improbable that cubic growth is due to modified sexual behavior. Hyman and Stanley [16] proposed a model (hereafter referred to as HSM) for HIV transmission within a homosexual community based on biased mixing; that is, the community is divided into categories consisting of individuals of similar risk behavior (where risk is quantified by the number of sexual partners), and it is assumed that individuals interact mainly with people of similar risk behavior. With this assumption, the magnitude of the epidemic, the expected time of its peak, and its growth rate all depend upon how different risk groups are distributed and how they interact with one another. Whereas the assumption of random mixing leads to the prediction that large numbers of low-risk individuals-that is, those who have relatively few new sexual partners per year-will become infected early on in the epidemic, biased mixing predicts an infection wave that passes from high-risk groups into those of lower risk. Colgate et al. [10] showed that a biased mixing model predicts cubic rather than exponential growth and hence is in accord with present data on AIDS cases.

The intuitive explanation for these quite different predictions is that with random mixing the infection tends to spread most rapidly in the most densely populated risk groups, which correspond to low-risk individuals. Biased mixing, however, assumes that high-risk individuals interact primarily among themselves; hence, they are the group who suffer most at the outset of the epidemic while lower risk individuals are not affected until the wave of infection has passed through intermediate risk groups. Hyman and Stanley [16] call this wave a "saturation wave," where saturation refers to the state of maximum infection in a given group. The term saturation is perhaps a little confusing as it suggests that no more individuals are infected after this point, which is not the case. We shall refer to this state as the state of maximum infection (SMI); that is, this is the point in a 
particular risk group when the number of newly infected individuals in that group is a maximum. After this point individuals continue to become infected but al a lesser rate.

One important consequence of this infection wave is that as it progresses into the lower risk groups, the intrinsic growth rate of the epidemic decreases proportionately so that rather than pure exponential growth the model predicts a polynomial growth rate in AIDS cases that can be approximated by a cubic [10,16]. Another consequence of the infection wave is that within a lower risk group the growth of HIV is rather complicated: At first, growth is exponential at a rate that corresponds to the intrinsic growth rate within this risk group, but as time progresses this intrinsic growth rate becomes dominated by the people infected from individuals in higher risk groups. Thus, one might predict that the total number of infected individuals at a given time could be approximated by a sum of exponentials (corresponding to risk groups far in front of the infection wave) and time-dependent exponentials that include both an intrinsic growth rate and interference from other risk groups. This interpretation differs from that of Colgate et al. [10].

A mathematical model that contains all factors that may contribute to the spread of AIDS would be very complex and impossible to solve analytically. Moreover, initial numerical simulation would provide little insight into the effect each factor has on the spread of infection and the interactions occurring between different factors. Consequently it is necessary, at first, to study simplified versions of the more general model that focus on particular aspects of the epidemic. In this paper we focus on biased mixing in a homosexual community, and we ignore such factors as variable infectiousness and variable incubation periods, which are accounted for in HSM. We stress that our model and analysis are not intended to give accurate predictions on the growth of the AIDS infection; rather, they are aimed at understanding how a particular assumption on biased mixing affects the spread of the disease. We show that although the total number of AIDS cases can be approximated very well with a polynomial, the mechanism according to which the disease is spreading may perhaps be better explained as a sum of various functions that differ significantly from polynomials.

In Section 2, we formulate a discrete model that is similar in many respects to the Hyman and Stanley continuous model with biased mixing but contains some simplifying assumptions. In Section 3, we present numerical simulations of our model to show that it captures the qualitative behavior of the more complex model. In Section 4 we derive expressions for the time to SMI in a particular risk compartment, the proportion of infected individuals at SMI, and the growth of infection up to SMI. Finally, in Section 5, we discuss our results. 


\section{MODEL EQUATIONS}

We restrict our study to the case of a homosexual community. We separate individuals into three general categories: uninfected susceptibles, infecteds without AIDS, and diagnosed AIDS cases. A susceptible person can become infected through sexual contact with an infected individual. We assume that movement from one category to another obeys the law of mass action. It is not known what fraction of individuals with HIV eventually proceed to develop AIDS. In this model, we assume this fraction to be 1, that is, that everyone with HIV will eventually develop AIDS. Those individuals who have AIDS are assumed to be removed from the population in the sense that they are no longer sexually active. We divide each of the three categories into $n$ different compartments, or activity classes, defined by risk behavior. Each compartment initially contains $1 / n$ of the total population under consideration. The risk behavior of each compartment is given by a mean risk defined as the average number of sexual partners per year (see [7] for a detailed discussion on different methods for quantifying activity within a particular class). Interaction within and between compartments is assumed to be homogeneous. There is a constant recruitment rate into all susceptible compartments that is assumed to be initially equal to the natural death rate so that the population is initially at equilibrium; this simplifies analysis and is a common assumption $[3,16]$.

We introduce the following definitions:

$$
\begin{aligned}
t & =\text { time (years) } \\
n & =\text { number of compartments }
\end{aligned}
$$

$S_{k}(t)=$ number of susceptibles in the $k$ th compartment at time $t$

$I_{k}(t)=$ number of infecteds in the $k$ th compartment at time $t$

$A_{k}(t)=$ number of AIDS cases in the $k$ th compartment at time $t$

$N_{k}(t)=$ total number of sexually active individuals in the $k$ th compartment at time $t$, that is $S_{k}(t)+I_{k}(t)$

$\beta=$ transmission efficiency per contact

$\gamma=$ rate at which infected individuals develop AIDS (per year)

$\mu=$ natural death rate (per year)

$\delta=$ death rate due to AIDS (per year)

$M_{k}=$ average number of sexual partners per year in the $k$ th compartment

$B_{k}=$ recruitment into the $k$ th compartment (per ycar)

$c(k, j)=$ average number of sexual contacts per partnership between people from compartments $k$ and $j$ 


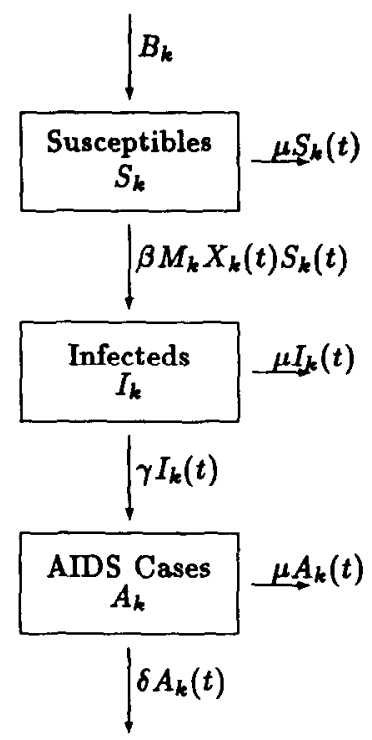

Fig. 1. Compartmental diagram for the $k$ th compartment of the model. The term $X_{k}(t)$ accounts for interaction with other compartments (see text for definitions).

$$
\begin{aligned}
p(t, k, j)= & \text { probability that a person in compartment } k \text { forms a } \\
& \text { partnership with a person in compartment } j \text { at time } t
\end{aligned}
$$

With these definitions, our model can be formulated as follows (see Figure 1 for compartmental diagram):

$$
\begin{aligned}
\frac{d S_{k}}{d t} & =B_{k}-\beta M_{k} X_{k}(t) S_{k}(t)-\mu S_{k}(t), \\
\frac{d I_{k}}{d t} & =\beta M_{k} X_{k}(t) S_{k}(t)-(\mu+\gamma) I_{k}(t), \\
\frac{d A_{k}}{d t} & =\gamma I_{k}(t)-(\mu+\delta) A_{k}(t)
\end{aligned}
$$

In the above equations, $X_{k}(t)$ is the expected number of contacts that an individual in the $k$ th compartment will have with infected individuals:

$$
X_{k}(t)=\sum_{j=1}^{n} p(t, k, j) c(k, j) \frac{I_{j}(t)}{N_{j}(t)} .
$$

We assume that high-risk individuals have approximately one contact per partnership but that the number of contacts per partnership increases for lower risk groups up to some maximum value. These assumptions may be captured by taking

$$
c(k, j)=1+c_{1} e^{-c_{2}\left(M_{k}+M_{j}\right)},
$$


where $c_{1}$ and $c_{2}$ are (positive) constants to be determined [16]. For our analytical proposes, only the qualitative form of $c(k, j)$ is important.

It is hypothesized that an individual infected with HIV undergoes an initial short period of high infectivity followed by a long period of low infectivity and then a period of high infectiousness immediately prior to developing AIDS [1]. (This hypothesis is based on virus in the blood, which may not be related to infectivity.) It also appears that the rate at which individuals develop AIDS varies with time from infection [10]. This suggests more complicated forms of $X_{i}(t)$ and of $\gamma$ that depend also on time from infection. For example, Jacquez et al. [17] model the long period to infectiousness prior to the onset of AIDS by a series of compartments that represent different stages of infection. However, to simplify analysis, we ignore this dependence. We also assume that people with AIDS die from the disease at a constant rate. The choices of $\gamma$ and $\delta$ correspond to means taken over observed rates.

It remains to define $p(t, k, j)$ in a way that accounts for biased mixing. In order to do this, we first need an interaction function, $f(r, s)$, which gives the frequency with which a person of risk $r$ forms a partnership with a person of risk $s$, where $r$ and $s$ are continuous variables corresponding to $M_{k}$. We simply use the interaction function given by Hyman and Stanley [16], namely,

$$
f(r, s)=\left[1+\frac{(r-s)^{m}}{\epsilon\left(r+r_{v}\right)^{m}}\right]^{-1},
$$

where we take $r_{v}=10, m=2$, and vary $\epsilon$ according to the degree of mixing we wish to allow between different risk groups. This function attains its maximum of 1 when $r=s$ and becomes very small when $r$ and $s$ are far apart, corresponding to the assumption that a person in risk group $r$ interacts mostly with others of similar risk. Using this frequency function, we can define our probability function, recalling that $M_{k}$ is the mean number of sexual partners in the $k$ th compartment, as

$$
p(t, k, i)= \begin{cases}\frac{f\left(M_{k}, M_{i}\right) M_{i} N_{i}(t)}{\sum_{j=1}^{n} f\left(M_{k}, M_{j}\right) M_{j} N_{j}(t)} & \text { if } i>k, \\ \frac{f\left(M_{i}, M_{k}\right) M_{i} N_{i}(t)}{\sum_{j=1}^{n} f\left(M_{i}, M_{j}\right) M_{j} N_{j}(t)} & \text { if } i<k, \\ 1-\sum_{j \neq k} p(t, k, j) & \text { if } i=k .\end{cases}
$$


We motivate the form of this function as follows. When $i>k$, we assume that the probability that an individual in the $k$ th compartment interacts with one in the $i$ th compartment is equal to the number of partnerships that a person in the $k$ th compartment forms with individuals in the $i$ th compartment, divided by the total number of partners that an individual in the $k$ th compartment has. When $i<k, p(t, k, i)$ must be adjusted because the total number of partnerships between the $k$ th and $i$ th compartments must equal the total number of partnerships between the $i$ th and $k$ th compartments; thus,

$$
M_{k} N_{k}(t) p(t, k, i)=M_{i} N_{i}(t) p(t, i, k) .
$$

Finally, $p(t, k, k)$ has been defined so that the probability of selecting a partner from the entire population is 1 . This function is the discrete analog of the continuous probability function, $\rho(t, r, s)$, used in HSM. Clearly one could assume different types of interaction function $f$ that would, in turn, lead to different probability functions. The particular $f$ chosen here is simply a quantitative example that captures the essential qualitative features of the assumptions on biased mixing. The paper by Stanley [20] discusses this in more detail.

\section{NUMERICAL RESULTS}

To illustrate the behavior of the model described by Equations (1)-(3), in our numerical simulations we choose parameter values and initial conditions similar to those used in HSM, namely, $\delta=0.5, \gamma=0.133, \mu=0.02$, $\beta=0.025, c(k, j)=1+10 e^{-0.1\left(M_{k}+M_{j}\right)}, B_{k}=\mu S_{k}(0), I_{k}(0)=10^{3} M_{k} / \sum_{j=1}^{n} M_{j}$, $A_{k}(0)=0$. The infected individuals are thus initially distributed according to risk so that at the outset of the epidemic there are more high-risk infecteds than low-risk (similar to HSM). This accounts for the observation that regardless of where the infection starts, it will rapidly move toward the higher risk groups. The qualitative nature of our numerical simulations is independent of the form of this initial condition. For the initial distribution of the population according to risk $r$ we use the inverse quartic given in $[10]$,

$$
N(r)=\frac{3 N_{0} \cdot 48^{3}}{(48+r)^{4}}
$$

and we take $N_{0}$, the total initial population, to be $10^{6}$. With this distribu- 
tion, the mean risk in compartment $k$ is given by

$$
M_{k}=\frac{\int_{r_{k}}^{r_{k+1}} r N(r) d r}{\int_{r_{k}}^{r_{k+1}} N(r) d r},
$$

where $r_{0}=0, r_{n}=\infty$, and $\int_{r_{k}}^{r_{k+1}} N(r) d r=N_{0} / n$.

In the simplest case, where $n=1$, our model essentially reduces to the simple homogeneous model formulated by Anderson and May [2], the primary difference being that we do not have a second class of infected individuals who do not go on to develop AIDS. In the next simplest case, $n=2$, we consider only two groups of people, low-risk and high-risk. In this case, the most important factor that affects qualitative behavior is the form of $f(r, s)$. Taking $\epsilon$ very small, there is only negligible interaction between low- and high-risk groups. One would thus expect two independent waves of infection that, initially, are exponential. Total growth of number of infected individuals in this case would be the sum of these exponentials (see Figure 2). As $\epsilon$ increases, $I_{2}(t)$ remains exponential but $I_{1}(t)$ becomes

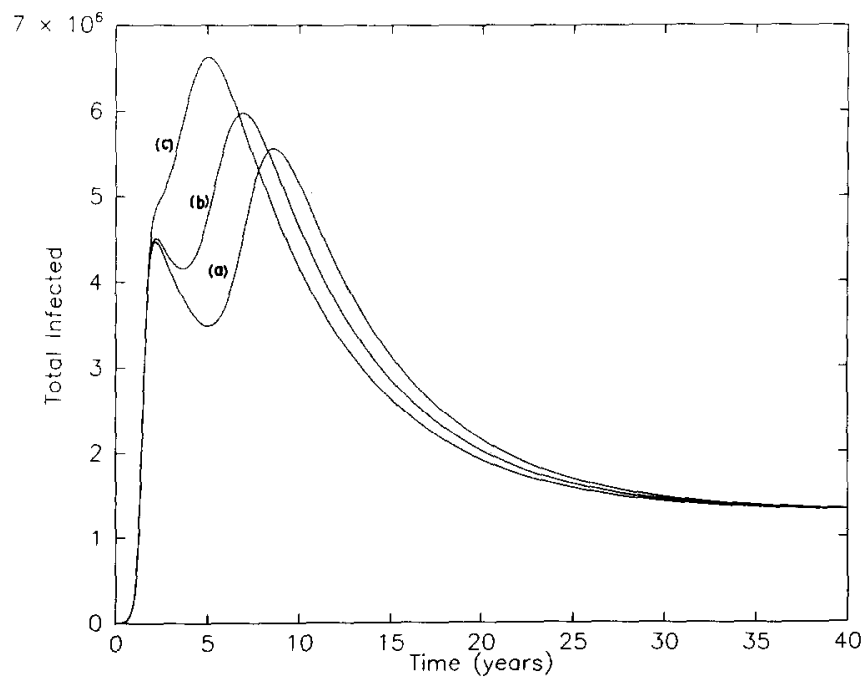

FIG. 2. Numerical solution of Equations (1)-(3) for two risk compartments. The time when the infection wave reaches the low-risk group depends heavily upon the rate of interaction between low-risk and high-risk groups. Here we show three different rates of interaction $f(r, s)$ : (a) $\epsilon=0.001$, (b) $\epsilon=0.01$, and (c) $\epsilon=0.1$. 


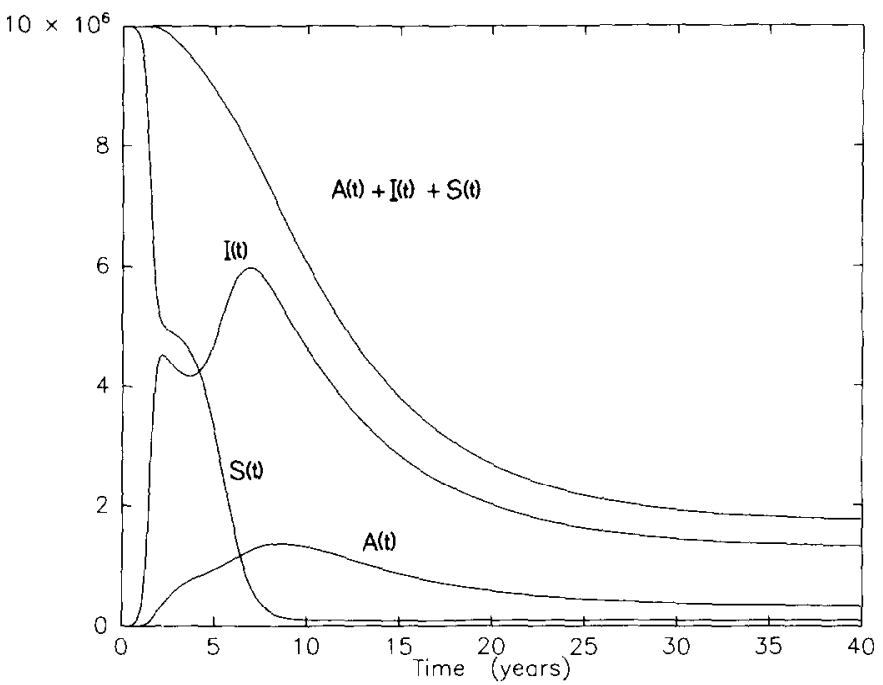

(a)

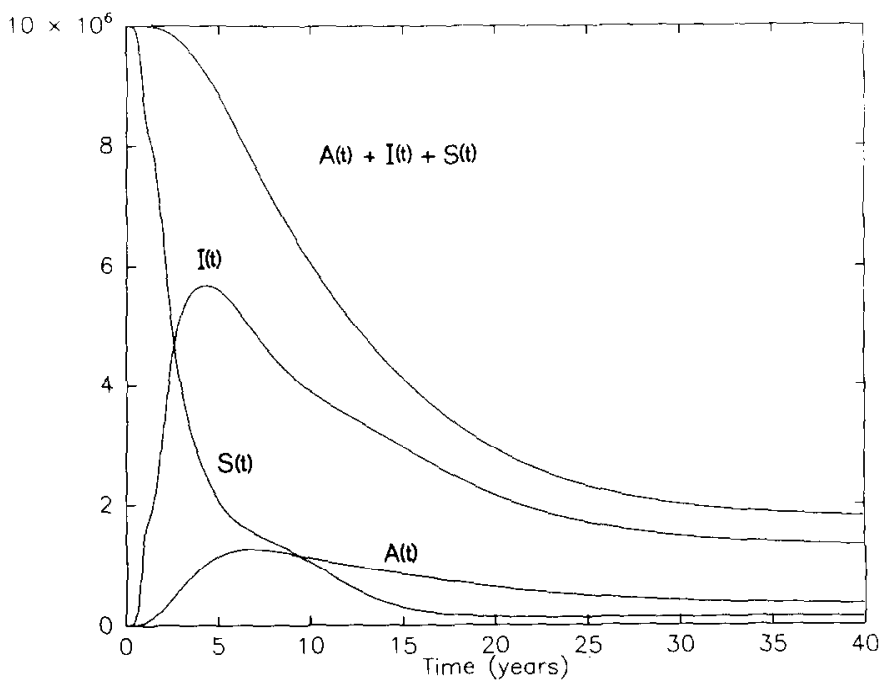

(b)

FIG. 3. (a) With only two compartments, the simple discrete model given by Equations (1)-(3) exhibits behavior that is qualitatively similar to that of the more complex HSM [16] and to the model of Jacquez et al. [17]. After a brief, initial exponential phase, the growth of the total number of AIDS cases, $A(t)$, is polynomial. The double-peak behavior of the infecteds, $I(t)$, occurs because we have only two risk groups with a weak interaction. By taking more risk groups, the effect of high-risk individuals on low-risk individuals is mediated through intermediate compartments smoothing out the curve of infection. (b) shows the behavior for six compartments. In both (a) and (b), $\epsilon=0.01$. $S(t)$ is the total number of susceptibles and $I(t)$ the total number of infecteds. 
increasingly distorted by interaction with the high-risk group. When $\epsilon$ is made large enough, the distortion is so great that the cumulative number of infecteds is smoothed out. This behavior is similar to that observed in the more complicated model of Jacquez et al. [17]. It is important to note that the two independent waves of infection in Figure $2 \mathrm{a}$ are a result of our discretization. This type of behavior would be observed in reality only if it were possible to identify two groups with different risk behavior that interacted only weakly. We note that even in the simplest case of two compartments we get behavior that is remarkably similar, at least qualitatively, to the predictions made by the more complicated HSM (see Figure 3). We also note that this serves to confirm that the rate at which the epidemic spreads and the shape of the infection wave depend heavily upon the rate of mixing between highly dissimilar groups $[10,15]$. When mixing is great, the infection wave approaches that predicted by the Anderson and May model in which partner selection is random.

With six compartments, one sees the infection wave predicted by HSM (see Figure 4). As was the case with the two-compartment model, the highest risk group initially exhibits exponential growth of infectives, which slows down prior to the group reaching SMI; in fact, we will see in the next

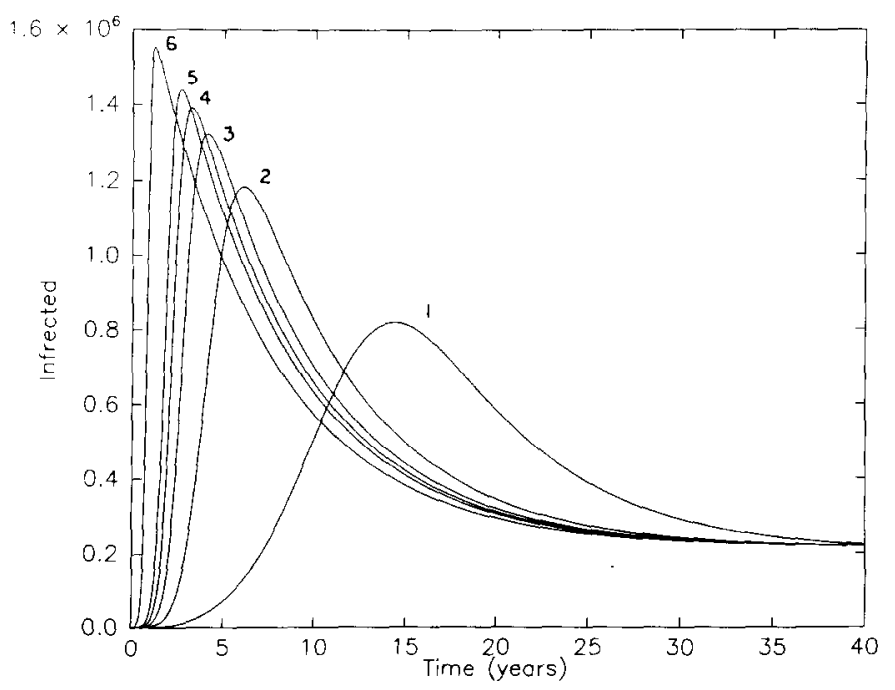

FIG. 4. Number of infected individuals in each risk group as a function of time. In this simulation we took six risk groups numbered from 6 (highest risk) to 1 (lowest risk), calculated as detailed in the text. We see the infection wave similar to that observed in HSM. High-risk groups become infected early on, and the epidemic slowly passes into lower risk groups. 
TABLE 1

Comparison of the Fraction $w_{k}$ of Individuals Infected at SMI in Each Risk Group $k$ Obtained from Numerically Solving the Full System Given by Equations (1)-(3) with the Bounds Calculated from Equation (14)*

\begin{tabular}{rcccc}
\hline$k$ & Risk $\left(M_{k}\right)$ & $w_{k}$ & Lower bound (14) & Upper bound (14) \\
\hline 1 & 9 & 0.7390 & 0.7221 & 0.7486 \\
2 & 28 & 0.7912 & 0.7583 & 0.8022 \\
3 & 47 & 0.8687 & 0.8337 & 0.8743 \\
4 & 66 & 0.9047 & 0.8659 & 0.9095 \\
5 & 84 & 0.9262 & 0.8818 & 0.9294 \\
6 & 103 & 0.9396 & 0.8913 & 0.9422 \\
7 & 122 & 0.9484 & 0.8994 & 0.9510 \\
8 & 140 & 0.9563 & 0.9112 & 0.9574 \\
9 & 161 & 0.9613 & 0.9315 & 0.9625 \\
10 & 190 & 0.9679 & 0.9545 & 0.9682 \\
11 & 248 & 0.9737 & 0.9721 & 0.9754 \\
12 & 599 & 0.9907 & 0.9897 & 0.9898 \\
\hline
\end{tabular}

${ }^{*}$ We estimate the amount of out-of-group mixing at SMI, $m_{k}$, by the initial out-of-group mixing.

section that up to SMI the growth of infectives for the highest risk compartment may be approximated by logistic growth. The other compartments have an intrinsic exponential growth that becomes dominated by the growth in higher risk compartments as they approach SMI. The total number of AIDS cases exhibits polynomial growth, similar to HSM. In each case, the greatest interference comes, as one might expect, from neighboring higher risk groups. Even when interaction rates between greatly differing risk groups are low, there may be no apparent delay in the total growth of infecteds, as there was in the two-compartment case, because the intermediate risk groups through which the infection wavc passes before it reaches the lowest risk individuals may smooth out the total infected curve. With a finer discretization (more compartments), the qualitative and quantitative differences are small, the main quantitative difference being that with many compartments the infection wave can reach low-risk individuals slightly sooner as a result of the introduction of a larger number of intermediate risk groups. We note that as risk decreases, the level of infection at SMI, measured by the fraction of people infected at SMI within a risk group, also decreases (see Table 1). This result is similar to that obtained by Jacquez et al. [17] with their more complicated model.

\section{ANALYSIS OF THE EPIDEMIC}

In this section we derive analytical expressions for the percentage of individuals infected in each risk compartment at SMI and the time to SMI. 
We first note that the model given by Equations (1)-(3) is unrealistic for people in very low risk compartments. We expect that such individuals would be engaged in long-term partnerships and that a pairing model of the type proposed by Dietz [12] would be more realistic. Therefore, the following analysis applies only to middle- and high-risk groups.

\subsection{FRACTION INFECTED IN EACH RISK COMPARTMENT AT SMI}

At the SMI time $t_{k}$ (or at steady state) in a given compartment $k$, $d I_{k} / d t=0$. We proceed to calculate bounds on the fraction of individuals infected in each particular risk group as follows. We define

$$
w_{k}=I_{k} / N_{k},
$$

where $I_{k}$ and $N_{k}$ are evaluated at SMI in risk category $k$. We let $m_{k}$ be the probability of out-of-group mixing for risk category $k$ at SMI; thus

$$
p\left(t_{k}, k, k\right)=1-m_{k}
$$

at SMI.

From the definition of $X_{k}$ and $c(k, j)$ and the observation that $w_{k} \leqslant 1$ for all $k$, the following inequalities are immediate:

$$
\left(1-m_{k}\right) c(k, k) w_{k} \leqslant X_{k} \leqslant c(k, 1) m_{k}+\left(1-m_{k}\right) c(k, k) w_{k} .
$$

Letting $\sigma_{k}=\left(1-m_{k}\right) c(k, k)$ and $\rho_{k}=c(k, 1) m_{k}$, Equation (10) becomes

$$
\sigma_{k} w_{k} \leqslant X_{k} \leqslant \rho_{k}+\sigma_{k} w_{k}
$$

At SMI in the compartment with risk $k, d I_{k} / d t=0$; hence, from Equation (2),

$$
w_{k}=X_{k} /\left(X_{k}+\alpha_{k}\right)
$$

where $\alpha_{k}=(\mu+\gamma) / \beta M_{k}$. Now the function $f(x)=x /(x+A)$ is an increasing function of $x$ for positive $A$; hence from Equation (11) we have

$$
\frac{\sigma_{k} w_{k}}{\sigma_{k} w_{k}+\alpha_{k}} \leqslant w_{k} \leqslant \frac{\rho_{k}+\sigma_{k} w_{k}}{\rho_{k}+\sigma_{k} w_{k}+\alpha_{k}} .
$$

From these inequalities we deduce the following bounds on $w_{k}$ :

$$
\max \left(0,1-\alpha_{k} / \sigma_{k}\right) \leqslant w_{k} \leqslant \lambda_{+}^{k},
$$

where $\lambda_{+}^{k}$ is the larger real root of the quadratic $\sigma_{k} x^{2}+\left(\rho_{k}+\alpha_{k}-\sigma_{k}\right) x-$ $\rho_{k}=0$. Note that if $m_{k}=0$, that is, there is no out of group mixing at SMI, 


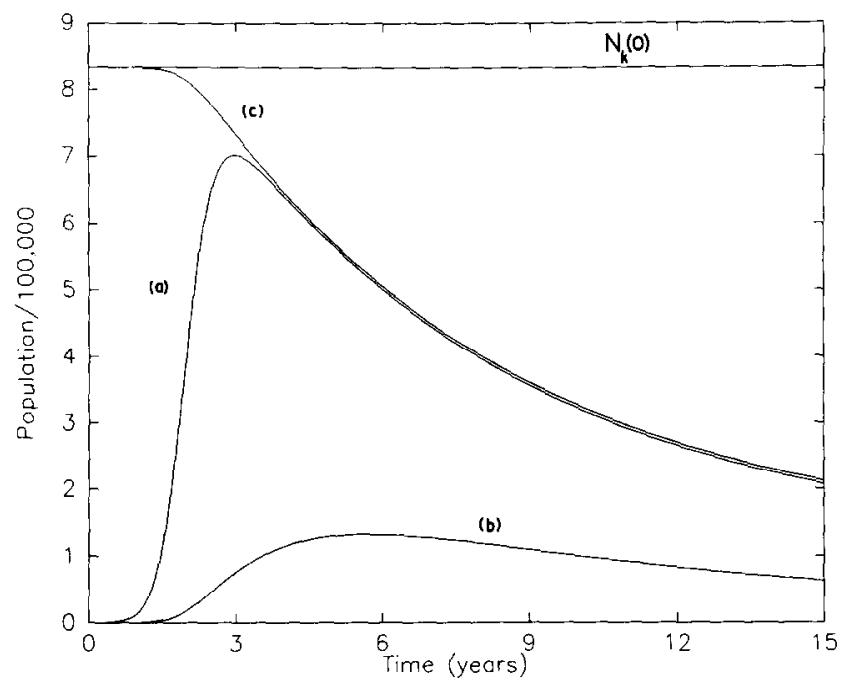

FIG. 5. Numerical simulation of Equations (1)-(3) with risk groups as in Table 1 showing (a) spread of infection, $I_{k}(t)$, (b) number of AIDS cases, $A_{k}(t)$, and (c) total number of individuals, $N_{k}(t)$, in each compartment. At SMI in the medium- and high-risk groups, the number of individuals in each group is roughly the same as before the infection began. That is, at SMI in medium- and high-risk groups, few people have developed AIDS. Also note that the ratio $I_{k} / N_{k}$ is approximately constant after SMI. Here we show the results for the risk group $k=9$. The qualitative form of the graph is similar for other high risk groups.

then $\rho_{k}=0$, which implies that the lower and upper bounds are equal and we have an exact value for $w_{k}$. Note that Equation (14) also holds at steady state for infection.

Clearly these bounds are very close together for small to medium out-of-group mixing, which will be the case from the form of the acceptance function $f$ given by Equation (6). Table I shows the values of $w_{k}$ obtained for a set of risk categories from simulating the full system given by Equations (1)-(3) together with the bounds calculated from the inequalities given in (14).

To calculate the number of infecteds in risk group $k$ at SMI we need to know the number of individuals $N_{k}\left(t_{k}\right)$ in that risk group at SMI. From curve (c) in Figure 5, it is clear that $N_{k}\left(t_{k}\right)$ is approximately equal to the initial population number, $N_{0} / n$, in high- and medium-risk groups. This observation implies that by the time SMI is reached in a particular compartment, the number of individuals who have gone on to develop AIDS in that compartment is small. Thus, the number of infectives in risk 
group $k$ at SMI can be estimated by

$$
I_{k}\left(t_{k}\right) \approx N_{0} \lambda_{+}^{k} / n
$$

In Appendix A, we show that this is an overestimate of the actual number of infectives at SMI in a particular risk group.

\subsection{THE TIME TO SMI}

We now find estimates on the time $t_{k}$ taken to reach SMI in the compartment with risk $M_{k}$. Assuming that at time $t_{k}$ the number of infectives in lower risk compartments is small (see Figure 4), Equation (2) can be approximated by

$$
\frac{d I_{k}}{d t}=\beta M_{k} \sum_{j=k}^{n} p(t, k, j) c(k, j) \frac{I_{j}(t)}{N_{j}(t)} S_{k}(t)-(\mu+\gamma) I_{k}(t)
$$

As we have noted above, this model is realistic only for middle- and high-risk groups; thus, $c(k, j) \approx 1$. As SMI approaches in a risk group $k$, we assume that higher risk groups arc at SMI or at stcady statc. Furthermore, our simulations suggest that the probability of interaction between two risk groups remains virtually constant, as does $N_{k}(t)$. Thus, Equation (16) may be approximated by

$$
\begin{aligned}
\frac{d I_{k}}{d t}= & \beta M_{k}\left[p(0, k, k) \frac{I_{k}(t)}{N_{k}(0)}+\sum_{j=k+1}^{n} p(0, k, j) w_{j}\right]\left[N_{k}(0)-I_{k}(t)\right] \\
& -(\mu+\gamma) I_{k}(t)
\end{aligned}
$$

which yields the solution

$$
I_{k}(t)=\frac{a_{1}+K a_{2} e^{b_{3} t}}{1+K e^{b_{3} t}}
$$

where

$$
\begin{aligned}
& b_{1}=\beta M_{k} p(0, k, k), \quad b_{2}=\beta M_{k} \sum_{j=k+1}^{n} p(0, k, j) w_{j}, \\
& b_{3}=\frac{b_{1}}{N_{k}(0)}\left(a_{2}-a_{1}\right), \quad K=\frac{I_{k}(0)-a_{1}}{a_{2}-I_{k}(0)}
\end{aligned}
$$




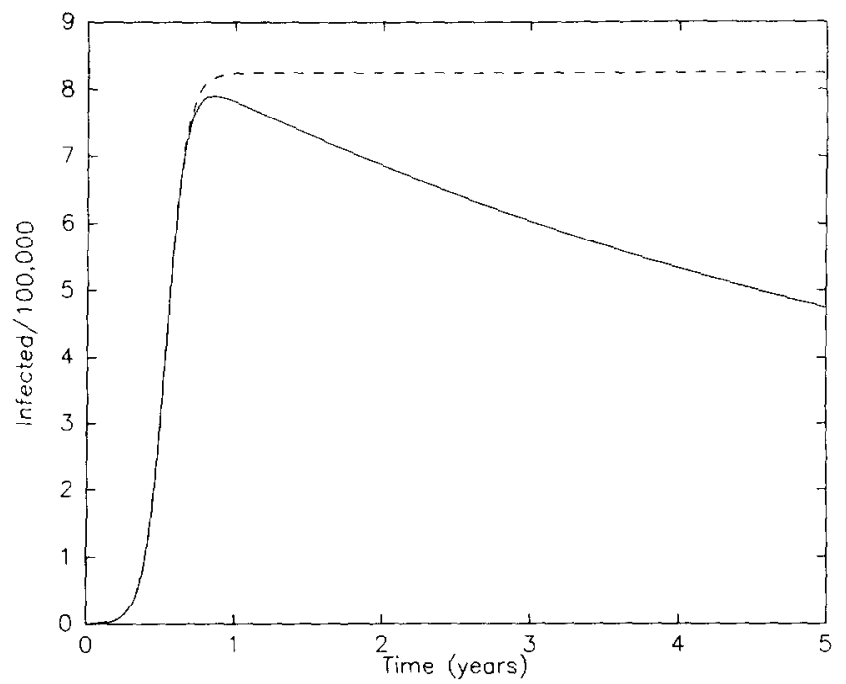

(a)

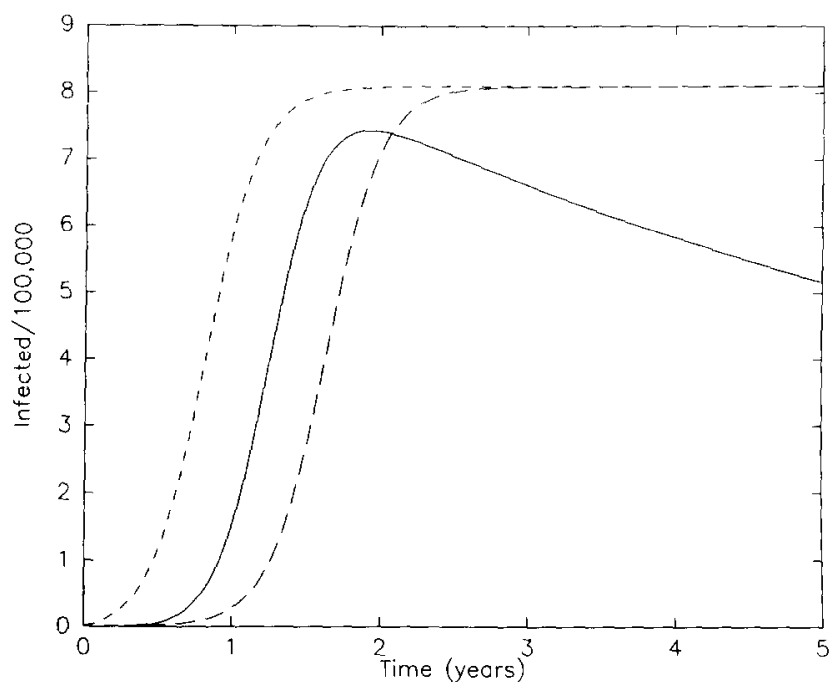

(b)

FiG. 6. (a) Logistic growth provides an excellent fit to the growth of number of infected individuals in the highest risk group up to SMI (see text for details). (Solid line) Actual number of infecteds in the highest risk group calculated from Equations (1)-(3). (Dashed line) Estimate of the number of infecteds for this risk group using the logistic growth approximation given by Equation (18). (b), (c) For risk groups $k=11$ and $k=10$, respectively, the solid line shows the number of infecteds as calculated from Equations (1)-(3); the short and long dashed lines show the logistic growth estimates using Equation (18) summing $j=k+1$ to $N$ and $k+2$ to $N$, respectively. These approximations enable us to estimate bounds for the time to SMI in high-risk groups (Table 2). 


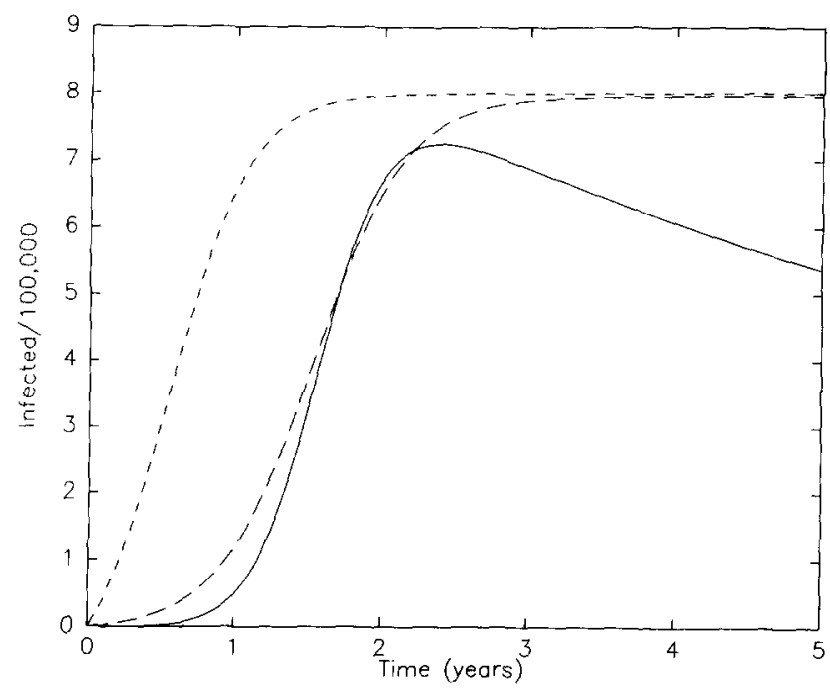

(c)

FIG. 6 (Continued)

and $a_{1}<0$ and $a_{2}>0$ are the roots of

$$
x^{2}+\frac{N_{k}(0)}{\bar{b}_{1}}\left(b_{2}+\mu+\gamma-b_{1}\right) x-N_{k}^{2}(0) \frac{b_{2}}{b_{1}}=0 .
$$

Equation (18), which is essentially the solution of a logistic growth equation, is a remarkably good approximation, for the highest risk group, to the actual growth of infectives (see Figure 6) up to a fraction $\phi$ of the carrying capacity $a_{2}$. As a group approaches its SMI it is clear that the logistic growth of Equation (17) will break down prior to SMI. This results from infected individuals in higher risk groups developing AIDS, thus decreasing the "growth" term in (16). Hence, the true value of $I_{k}(t)$ never reaches $a_{2}$ as Equation (18) suggests. As a result we estimate the time to SMI as

$$
t_{k}=\frac{1}{b_{3}} \log \left[\frac{\phi a_{2}-a_{1}}{K a_{2}(1-\phi)}\right] .
$$

Note that in the above analysis the $w_{j}$ 's are unknown. Since we are assuming that the higher risk groups are at steady state or at SMI, we may approximate $w_{j}$ by $\lambda_{+}^{j}$ (from Table 1). Furthermore, although we cannot find an a priori value for $\phi$, our numerical simulations show that the logistic growth curve approximates the actual growth up to a very large 
TABLE 2

Comparison of Time to SMI, $t_{k}$, from Equation (19) with the Actual SMI Time for High-Risk Groups*

\begin{tabular}{cccc}
\hline$k$ & $t_{k}(\operatorname{sum} k+1$ to $N)$ & $t_{k}(\operatorname{sum} k+2$ to $N)$ & Actual $t_{k}$ \\
\hline 9 & 2.31 & 3.64 & 2.76 \\
10 & 2.01 & 3.16 & 2.46 \\
11 & 1.80 & 2.62 & 1.98 \\
12 & 0.89 & 0.89 & 0.88 \\
\hline
\end{tabular}

${ }^{*}$ See text for details.

fraction of the carrying capacity $a_{2}$ and that Equation (19) is reasonably insensitive to the actual value of $\phi$ for large values. Table 2 compares this estimate for a typical value of $\phi$ with the actual SMI time for the high-risk groups.

Figure 6 and Table 2 show that the above approximation leads to an excellent fit for the highest risk group. However, Table 2 shows that for groups with risk lower than the highest, the predicted SMI time is much less than the actual SMI time. Furthermore, the approximation to the growth of infection given by Equation (18) grows too quickly. This is to be

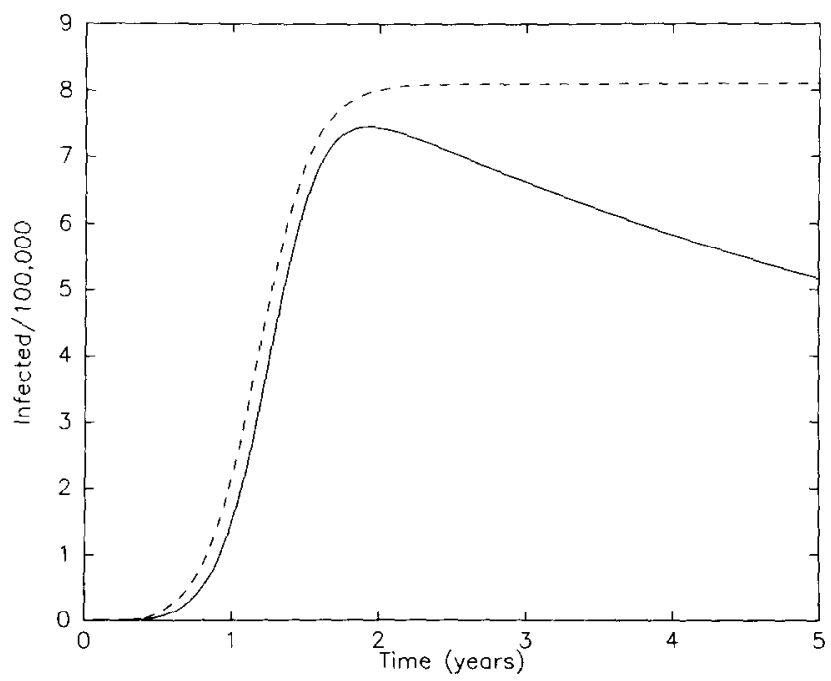

FIG. 7. Estimate of growth of number of infected individuals in the risk group given by $k=11$ using the two-time-scale procedure outlined in Appendix B. The dashed line is the approximation. 


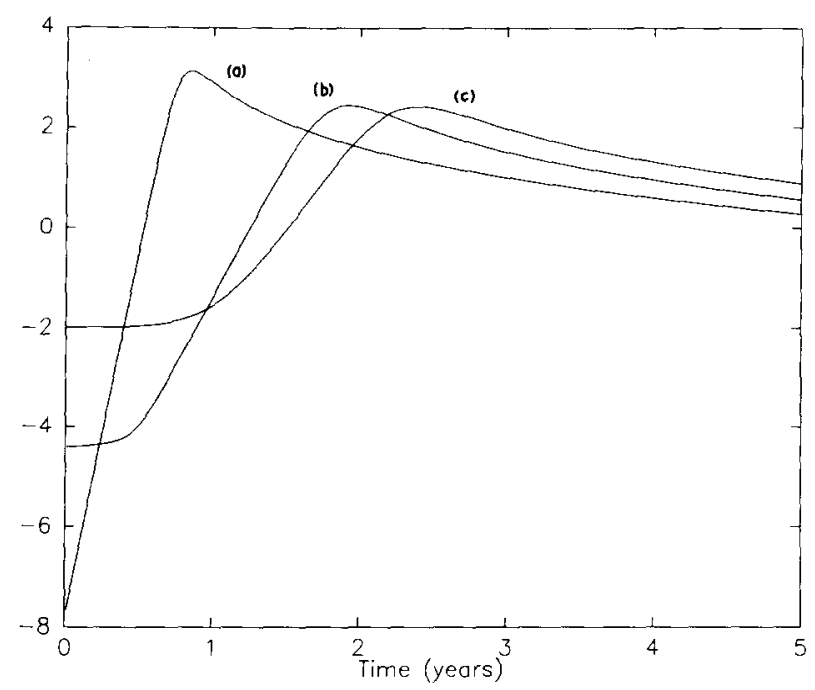

FIG. 8. Plot of $\ln \left[\left(I_{k}-a_{1}\right) / K\left(a_{2}-I_{k}\right)\right]$ against time for (a) $k=12$, (b) $k=11$, and (c) $k=10$, showing that high-risk groups exhibit logistic growth as they approach SMI and that during this phase of growth the logistic growth approximation given by Equation (18) is a good one.

expected because in Equation (17) we assume that every compartment above the one in question has reached SMI or steady state. This is true for compartments of risk much higher than that in question, but not for the immediate higher risk group, in which the infection is still growing. Thus the sum from $j=k+1$ to $N$ in Equation (17) is an overestimate of the interaction that compartment $k$ experiences with infecteds from higher risk groups. For high-risk groups, therefore, we get a lower bound on the time to SMI. Summing from $j=k+2$ to $N$ would give us an upper bound for the time to SMI for high-risk groups (see Table 2 and Figure $6 \mathrm{~b}, \mathrm{c}$ ). Note that for lower risk groups it is impossible to say a priori whether the above predictions are upper or lower bounds because other factors, such as death due to AIDS in the very high risk groups, come into play.

In Appendix B we outline a multi-time-scale procedure to approximate the growth to SMI of infecteds in high-risk groups (Figure 7). Figure 8 shows that the number of infecteds in high-risk groups exhibits logistic growth just prior to SMI.

\section{DISCUSSION}

We have analyzed the growth of HIV in a simplified version of the model presented by Hyman and Stanley [15] for a homosexual community 
wherein sexual behavior is assumed to be heterogeneous. Because of the simplifying assumptions we make, our model cannot give accurate predictions for the spread of HIV infection. This is also true of the more complicated models. However, the simple form of our model captures much of the qualitative behavior of more complex models and enables us to quantitatively analyze the spread of infection through different risk groups and to investigate the dynamics of the spread from one risk group to another. Moreover, we can calculate upper and lower bounds for the fraction infected at SMI and the time to SMI in high-risk groups in terms of the parameters of the model. For example, Equation (14) shows that the fraction of individuals infected at SMI in a particular risk group increases as out-of-group mixing increases or as the average number of sexual partners increases. Our analysis shows that the growth of HIV infection appears to be composed of several parts. Initially, when the infection wave is passing through the highest risk group, growth of the infection is approximately logistic, but as it reaches high- and middle-risk groups, interference begins to play a large role and complicates behavior. We present a two-time-scale procedure to analyze this case and show that growth of infection in middle- and high-risk groups may be closely approximated by an initial exponential phase followed by a logistic growth phase up to SMI.

One important aspect of risk-based models is the quantification of risk. In both our model and HSM there is no difference in intrinsic growth rates between a group of individuals who have 50 contacts with one partner over a year and a group who have one contact with 50 different individuals. Yet there clearly ought to be a difference in the way infection spreads in such groups. In such cases we need to use a model similar to that proposed by Dietz [12], which takes into account the duration of relationships. Thus we restrict the analysis of our model to that of middle- and high-risk individuals. Low-risk behavior should be modeled as in [12]. Furthermore, contact patterns are also important. Jacquez et al. [17] presented a detailed numerical analysis of the effects of different contact patterns. Recently, Gupta et al. [13] analyzed the significance of the sexual contact network by studying a number of cases ranging from the extremes of high within-class contact to low within-class contact. Anderson et al. [5] considered the case of movement between sexual activity classes as a result of change in behavior. In this paper we have presented a model that assumes one particular type of contact pattern and, in terms of risk-based behavior, is appropriate for middle- and high-risk groups. The analysis presented here is a first step in analyzing more complex behavior patterns.

In this paper we have used the abbreviation SMI to describe the state of a risk compartment when it reaches maximum infection. This terminology contrasts with that of Hyman and Stanley. Their terminology may be 
misleading, for the term saturation usually means that a certain carrying capacity has been attained and that the system will remain there for some time. A more appropriate, albeit more cumbersome, term is state of maximum infection, which we employ here.

This work was started while $M . N$. was attending an NSF-funded REU program, grant number NSF DMS 8900490, at the Department of Mathematics, University of Utah, during the summer quarter of 1989. G. D. Y. would like to acknowledge NIH grant \#GM29123-09, and to thank the Department of Mathematics, University of Utah, for financial support.

\section{APPENDIX A. NUMBER OF INFECTIVE INDIVIDUALS AT SMI}

Here we show that Equation (15) is an overestimate of the number of individuals infected in a particular risk group at SMI in that risk group. We show, in fact, that (i) $\lambda_{+}^{k}\left(m_{k}(0)\right) \geqslant \lambda_{+}^{k}\left(m_{k}\left(t_{k}\right)\right)$ and (ii) $N_{k}(0) \geqslant N_{k}\left(t_{k}\right)$.

To show (i) we first recall that $\lambda_{+}^{k}$ is the larger root of the quadratic $\sigma_{k} x^{2}+\left(\rho_{k}+\alpha_{k}-\sigma_{k}\right) x-\rho_{k}=0$, where $\sigma_{k}, \rho_{k}$, and $\alpha_{k}$ are defined in the text. Differentiating this quadratic with respect to $m$, the mixing, we have

$$
\frac{d x}{d m}=\frac{[c(k, k) x-c(k, 1)](x-1)}{(2 x-1) \sigma_{k}+\rho_{k}+\alpha_{k}}
$$

As the model is realistic only for medium- and high-risk groups, we may approximate both $c(k, k)$ and $c(k, 1)$ by 1 . Thus, Equation $(\Lambda .1)$ becomes

$$
\frac{d x}{d m}=\frac{(x-1)^{2}}{(2 x-1) \sigma_{k}+\rho_{k}+\alpha_{k}},
$$

and, clearly,

$$
\frac{d x}{d m} \geqslant 0 \quad \text { for } x \geqslant \frac{1}{2}
$$

Our numerical simulations show that $m_{k}\left(t_{k}\right) \leqslant m_{k}(0)$ and that $\lambda_{k}^{+}>1 / 2$. Therefore, Equation (A.3) shows that $\lambda_{k}^{+}\left(m_{k}(0)\right) \geqslant \lambda_{k}^{+}\left(m_{k}\left(t_{k}\right)\right)$.

To show (ii), we add Equations (1) and (2) to get

$$
\frac{d N_{k}}{d t}=\mu\left[N_{k}(0)-N_{k}(t)\right]-\mu I_{k}(0)-\gamma I_{k}(t) .
$$

Clearly, $d N_{k} / d t \leqslant 0$ at $N_{k}(t)=N_{k}(0)$; hence $N_{k}\left(t_{k}\right) \leqslant N_{k}(0)$.

\section{APPENDIX B. TIME-SCALE MATCHING}

As shown in Figure 6 and Table 2, the approximation in Equation (17) is good for the highest risk group but is poor for lower risk groups. This is 
because the approximation made in Equation (17) assumes that during the whole course of infection in a particular risk group, all higher risk groups are at SMI or steady state. Although this is a good approximation for the latter stages of infection in a particular risk group, it is invalid for the initial stage. During the early stages of infection in a medium- or high-risk group, its neighboring groups will also be in early stages of infection. Thus, a better approximation to the growth of infection in a risk group $k$ during the early stages would be to put $b_{2}=0$ in Equation (18), that is, assume that there is no infection from other risk groups. The approximation to the initial growth of infection is thus given by Equation (18) with $b_{2}=0$. In order to get a solution up to SMI, one has to match this to the solution of the more general form of Equation (18) with $b_{2}$ nonzero where, in the latter, one has to replace $I_{k}(0)$ with the value of $I_{k}$ at the matching time. Figure 7 shows the result of this procedure. The matching time, $t_{m}$, was taken to be the time at which $I_{k}=10^{3}$, that is,

$$
t_{m}=\frac{1}{a} \ln \frac{10^{3}}{I_{k}(0)} .
$$

Note that although we approximate the initial phase of infection by logistic growth, because the number of infected individuals is small this is approximately exponential.

Although crude, the above procedure proved to be reasonably accurate for estimating $I_{k}(t)$ for high-risk groups. The procedure assumes that there are two phases for infection up to SMI: an initial exponential growth phase in which the group acts mainly within itself, and a later logistic growth phase in which the group interacts with higher risk groups all of which are at SMI or steady state. A numerical study of the medium- and high-risk groups shows that they do exhibit logistic growth toward SMI (Figure 8). A more refined scheme would take account of the transition phase, wherein the interaction of the group with higher risk groups changes from zero to that appropriate at SMI. This would require detailed information that could be obtained only from the simulations. As this transition phase is rather short, it appears that ignoring it can still give good approximations.

\section{REFERENCES}

1 R. M. Anderson, The epidemiology of HIV infection: variable incubation plus infectious periods and heterogeneity in sexual activity, J. Roy. Statist. Soc. A, 151:66-93 (1988).

2 R. M. Anderson and R. M. May, The invasion, persistence and spread of infectious diseases within animal and plant communities, Phil. Trans. R. Soc. Lond. B 314:533 -570 (1986). 
3 R. M. Anderson, R. M. May, G. F. Medley, and A. Johnson, A preliminary study of the transmission dynamics of the human immunodeficiency virus (HIV), the causative agent of AIDS, IMA J. Appl. Med. Biol. 3:229-263 (1986).

4 R. M. Anderson, R. M. May, and A. R. McLean, Possible demographic consequences of AIDS in developing countries, Nature (Lond.) 332:228-234 (1988).

5 R. M. Anderson, S. P. Blythe, S. Gupta, and E. Konings. The transmission dynamics of the human immunodeficiency virus type 1 in the male homosexual community in the United Kingdom: the influence of changes in sexual behaviour, Phil. Trans. Roy. Soc. B 325:45-98 (1989).

6 R. M. Anderson, T. W. Ng, M. C. Boily, and R. M. May, The influence of different sexual contact patterns between age classes on the predicted demographic impacts of AIDS in developing countries, Ann. N.Y. Acad. Sci. 569:240-274 (1989).

7 S. P. Blythe and R. M. Anderson, Heterogeneous sexual activity models of HIV transmission in male homosexual populations, IMA J. Math. Appl. Med. Biol. 5:237-260 (1988).

8 C. Castillo-Chavez, K. Cooke, W. Huang, and S. A. Levin, On the role of long incubation periods in the dynamics of acquired immunodeficiency syndrome (AIDS). Part 1: Single population models, J. Math. Biol. (1989), 27, 373-398.

9 S. A. Colgate, J. M. Hyman, and E. A. Stanley, A risk-based model explaining the cubic growth in AIDS cases, (Los Alamos Natl. Lab., Los Alamos, NM), Rep. LAUR 87-3412, 1987.

10 A. Colgate, E. A. Stanley, J. M. Hyman, S. P. Layne, and C. Qualls, Risk behaviorbased model of the cubic growth of acquired immunodeficiency syndrome in the United States, Proc. Natl. Acad. Sci. USA 86:4793-4797 (1989).

11 Centers for Disease Control, AIDS Public Information Data Set AIDS07, Centers for Disease Control, Atlanta, GA., 1987.

12 K. Dietz, On the Iransmission dynamics of HIV., Math. Biosci., 90:397-414 (1988).

13 S. Gupta, R. M. Anderson, and R. M. May, Networks of sexual contacts: implications for the pattern of spread of HIV, AIDS 3:807-817 (1989).

14 H. W. Hethcote, A model for HIV transmission and AIDS, in Mathematical Approaches to Problems in Resource Management and Epidemiology, Springer-Verlag, Berlin, 1989, eds. C. Castillo-Chavez, S. A. Levin, C. A. Shoemaker, pp. 164-176.

15 J. M. Hyman and E. A. Stanley, Using mathematical models to understand the AIDS epidemic, Math. Biosci. 90:415-474 (1988).

16 J. M. Hyman and E. A. Stanley, The effect of social mixing patterns on the spread of AIDS, in Mathematical Approaches to Problems in Resource Management and Epidemiology, Lecture Notes in Biomathematics, Springer-Verlag, Berlin, 1989, eds. C. Castillo-Chavez, S. A. Levin, C. A. Shoemaker, pp. 190-219.

17 J. A. Jacquez, C. P. Simon, J. Koopman, L. Sattenspiel, and T. Perry, Modelling and analyzing HIV transmission: the effect of contact patterns, Math. Biosci. 92:119-199 (1988).

18 R. M. May and R. M. Anderson, Transmission dynamics of HIV infection, Nature 326:137-142 (1987).

19 R. M. May and R. M. Anderson, The transmission dynamics of human immunodeficiency virus (HIV), Phil. Trans. Roy. Soc. B 321:565-607 (1989).

20 E. A. Stanley, Mathematical models of the AIDS epidemic: an historical perspective, in Lectures in Complexity, Santa Fe Institute Studies in the Science of Complexity, Reading, MA: Addison-Wesley, Vol. VIII 1989. 